\title{
CERTAIN LINEAR COMBINATIONS OF HAUSDORFF SUMMABILITY METHODS
}

\author{
BY \\ B. VANDERBURG
}

1. Introduction. Throughout, $R_{\epsilon}$ denotes the half-plane $R(z)>-\epsilon . \epsilon^{\prime}$ and $\epsilon$ are always assumed to satisfy the relation $0<\epsilon^{\prime}<\epsilon<1$.

We shall be concerned with Hausdorff summability methods $\left({ }^{1}\right)$ whose generating sequences are of the form $\{T(n)\}_{0}^{\infty}$, where $T(z)$ is a regular function on some $R_{\epsilon}$. If $T_{1}(z), T_{2}(z)$ are two such functions, we know the following to be true $\left({ }^{2}\right)$ : (i) If $\left[T_{1}(z) / T_{2}(z)\right]$ is a Mellin transform (pure, in the sense of Hausdorff [4]), that is,

$$
T_{1}(z) / T_{2}(z)=\int_{0}^{1} x^{z} d \phi(x)
$$

where $\phi(x)$ is of b.v. on $(0,1)$ and $\phi(0)=\phi(0+)$, then every sequence limitable- $H\left(T_{2}\right)$ to $s$ is limitable- $H\left(T_{1}\right)$ to $T_{1}(0) s / T_{2}(0)$. When this condition is fulfilled, we say $H\left(T_{1}\right)$ is as strong as $H\left(T_{2}\right)$, and write this $H\left(T_{1}\right) \supset H\left(T_{2}\right)$. (ii) If $T_{1}(0) / T_{2}(0)=1$, and both $\left[T_{1}(z) / T_{2}(z)\right]$ and its reciprocal are Mellin transforms, then a sequence is $H\left(T_{1}\right)$-convergent to a limit $s$, only if it is $H\left(T_{2}\right)$-convergent to $s$, and conversely. If this latter condition holds, the methods are called equivalent.

The following is true $[1 ; 6 ; 7]:$ If on $R_{\epsilon}, T(z)$ is regular, and

$$
\int_{0}^{1}|T(x+i y)|^{2} d y<c<+\infty
$$

uniformly, then there exists a function $\phi(x)$, which is a.c. on $(0,1)$ and such that

$$
T(z)=\int_{0}^{1} \phi^{\prime}(x) x^{z} d x
$$

on the half-plane $\Re(z)>\epsilon$.

From this we obtain the following: (1) Suppose on $R_{\epsilon}, T(z)$ is regular and further

Presented to the Society, November 26, 1949, under the title $A$ linear combination of Cesàro and Hölder summability methods; received by the editors May 1, 1950 and, in revised form, December 11, 1950.

(1) The methods are not necessarily as powerful as ordinary convergence.

(2) For classical results in this field, the reader is referred to $[2 ; 4 ; 9 ;$ and 11$]$; more recent results are in $[1 ; 7]$. Numbers in brackets refer to the bibliography at the end of the paper. 


$$
T(z)=a+O\left(|z+1|^{-1}\right),
$$

uniformly. Then $T(z)$ is a Mellin transform. This is an immediate result since $(T(z)-a)$ satisfies (1.2). (2) If $T(z)$ satisfies the conditions of (1) $(a \neq 0)$, then $T(z)^{-1}$ is a Mellin transform if, and only if, it is free from zeros on $R_{0}$. The necessity of the condition is obvious. To show that it is sufficient, we note that

$$
T(z)^{-1}=a^{-1}+O\left(|1+z|^{-1}\right),
$$

uniformly on $R_{\mathrm{e}}$. For some value $\rho>0$, it is then true that $T(z) \neq 0$ on $R_{\mathrm{e}}$ provided $|\Im(z)| \geqq \rho$. By the Heine-Borel theorem, it follows that there exists an $\epsilon^{\prime} \leqq \epsilon$, such that $T(z) \neq 0$ in the rectangle $|\Im(z)| \leqq \rho,-\epsilon^{\prime} \leqq \Re(z) \leqq 0$. Hence $T^{-1}(z)$ satisfies the hypotheses of (1) with $\epsilon$ replaced by $\epsilon^{\prime}$.

The summability methods of Cesàro and Hölder, $C^{\alpha}$ and $H^{\alpha}$, are associated with the functions

$$
\begin{aligned}
& C^{\alpha}(z)=\Gamma(\alpha+1) \Gamma(z+1) / \Gamma(\alpha+z+1), \\
& H^{\alpha}(z)=(z+1)^{-\alpha} .
\end{aligned}
$$

We have:

(A) Let $\alpha$ be any real or complex number. If $T(z)$ is regular on some $R_{e}$, and

$$
T(z)=a(z+1)^{-\alpha}+O\left(|z+1|^{-\alpha-1}\right)
$$

uniformly on $R_{\mathrm{e}}$, then $H(T) \supset H^{\alpha}$.

(B) If the hypotheses of (A) are true $(a \neq 0)$, then $H^{\alpha} \supset H(T)$ if, and only if, $T(z) \neq 0$ on $R_{0}$.

These results follow at once from an application of (i), (1), and (2). We may replace $H^{\alpha}$ by $C^{\alpha}$ in both statements if $\Re(\alpha)>-1$. This follows from the equivalence of the two methods under these circumstances $[2 ; 4]$.

We need the following [10]: Suppose $n$ is any positive integer, and $\alpha$ is any complex number. Then

$$
\begin{aligned}
\frac{\Gamma(z+1)}{\Gamma(\alpha+z+1)}= & \frac{1}{(z+1)^{\alpha}}+\frac{\alpha \lambda_{1}(\alpha)}{(z+1)^{\alpha+1}+\cdots} \\
& +\frac{\alpha(\alpha+1) \cdots(\alpha+n-1) \lambda_{n}(\alpha)}{(z+1)^{\alpha+n}} \\
& +O\left(|z+1|^{-\alpha-n-1}\right) .
\end{aligned}
$$

This holds uniformly on $R_{c}, c<+\infty$. The values $\lambda_{1}(\alpha), \cdots, \lambda_{n}(\alpha)$ are chosen so that

$$
\begin{gathered}
(1-x)^{\alpha-1}-\left(\log \frac{1}{x}\right)^{\alpha-1}-\cdots-\lambda_{n}(\alpha)\left(\log \frac{1}{x}\right)^{\alpha+n-1} \\
=f(x)(1-x)^{\alpha+n}
\end{gathered}
$$


where $f(x)$ is regular at $x=1$. For example, $\lambda_{1}(\alpha)=(1-\alpha) / 2$.

2. The summability method $\left[C^{\alpha}-\Gamma(\alpha+1) H^{\alpha}\right]$. Consider the function

$$
T(z)=C^{\alpha}(z) / \Gamma(\alpha+1)-H^{\alpha}(z) .
$$

From (1.9) it is seen that for some $\epsilon, 0<\epsilon<1$,

$$
T(z)=\frac{\alpha(1-\alpha)}{2(z+1)^{\alpha+1}}+O\left(|z+1|^{-\alpha-2}\right) .
$$

Thus, by statement (A), $\S 1, H(T) \supset H^{\alpha+1}$ for all values of $\alpha$. In a slightly different form, we have $H(\Gamma(\alpha+1) T)$, written $\left[C^{\alpha}-\Gamma(\alpha+1) H^{\alpha}\right] \supset H^{\alpha+1}$. $(\alpha \neq-1,-2, \cdots)$.

As for the converse of this result, we shall confine ourselves to the real regular case. Thus, throughout, $\alpha$ is supposed real and to exceed -1 . We can write (2.1) in the form

$$
T(z)=\frac{\Gamma(z+1)}{\Gamma(\alpha+z+1)}\left[1-\frac{\Gamma(\alpha+z+1)}{(z+1)^{\alpha} \Gamma(z+1)}\right] .
$$

It is seen that $T(z)=0$ if, and only if,

$$
F(z, \alpha)=\Gamma(\alpha+z+1) /(z+1)^{\alpha} \Gamma(z+1)=1 .
$$

First, suppose that $z(=x)$ is real and not less than 0 . Then (2.4) is true only if $\alpha=0$ or $\alpha=1$. For it is known, and may easily be proven by (2.7) to follow, that $F(\alpha+x+1)$ is a logarithmically convex function of $\alpha$. Hence it cannot take on the value 1 more than twice.

We now suppose $z=x+i y, y \neq 0$. Concerning $F(x+i y, \alpha)$ the following is true.

Lemma 1. If $y \neq 0$, the function $\log |F(x+i y, \alpha)|$ is, as a function of $\alpha$, concave at $\alpha=-x-1$. It decreases steadily as $\alpha$ varies from $-x-1$ to $-x$, and changes from concave to convex at some point between these two values (but less than $|y|-x-1)$. Thereafter it is convex for all values of $\alpha$.

Momentarily assuming the truth of this, we have the following: Since $z=x+i y, y \neq 0$, by the lemma, $\log |F(x+i y, \alpha)|$ decreases as $\alpha$ varies from $-x-1$ to $-x$. Thereafter the function is convex. Since it is zero at $\alpha=0$ $(\geqq-x)$ and $\alpha=1$, it is seen that it must be positive on the open interval $(-x-1,-x)$. Thus $F(x+i y, \alpha)=1$ only if $\alpha=0$ or 1 . We have then by statement (B), $\$ 1$, and the opening result of this section:

I. At least when they are both defined and are real and regular, the methods $H^{\alpha+1},\left[C^{\alpha}-\Gamma(\alpha+1) H^{\alpha}\right][1-\Gamma(\alpha+1)]^{-1}$ are equivalent.

Proof of Lemma 1. It is seen from (1.9) that on $R_{\epsilon}$

$$
\lim _{|z| \rightarrow \infty} F(z, \alpha)=1 \text {. }
$$


The functional equation of the gamma function now yields

$$
F(z, \alpha)=\left(\frac{z+2}{z+1}\right)^{\alpha}\left(\frac{z+\alpha+1}{z+1}\right) F(z+1, \alpha) .
$$

It follows from this and (2.5) that

$$
F(z, \alpha)=\prod_{0}^{\infty}\left[\frac{(z+j+2)^{\alpha}(z+j+1)}{(z+j+1)^{\alpha}(z+\alpha+j+1)}\right] .
$$

Thus we have

$$
|F(z, \alpha)|^{2}=\prod_{0}^{\infty}\left[\frac{(x+j+2)^{2}+y^{2}}{(x+j+1)^{2}+y^{2}}\right]^{\alpha}\left[\frac{(x+j+1)^{2}+y^{2}}{(x+\alpha+j+1)^{2}+y^{2}}\right]
$$

$$
2 \log |F(z, \alpha)|=\sum_{0}^{\infty}\left\{\alpha \int_{0}^{x+j+2} \frac{2 t d t}{t^{2}+y^{2}}-\int_{x+j+1}^{\alpha+x+j+1} \frac{2 t d t}{t^{2}+y^{2}}\right\} ;
$$

0) $2 \frac{\partial}{\partial \alpha} \log |F(z, \alpha)|=\sum_{0}^{\infty}\left\{\int_{x+j+1}^{x+j+2} \frac{2 t d t}{t^{2}+y^{2}}-\frac{2(\alpha+x+j+1)}{(\alpha+x+j+1)^{2}+y^{2}}\right\}$;

$$
\frac{\partial^{2}}{\partial \alpha^{2}} \log |F(z, \alpha)|=\sum_{0}^{\infty} \frac{(\alpha+x+j+1)\left[3 y^{2}-(\alpha+x+j+1)^{2}\right]}{\left[(\alpha+x+j+1)^{2}+y^{2}\right]^{3}} .
$$

Lemma 1 is now seen to be an immediate consequence of the following:

(a) $\log |F(z,-x-1)|>\log |F(z,-x)|$.

(b) The graph of $\log |F(z, \alpha)|$ has, as a function of $\alpha$, parallel tangents at $\alpha=-x$ and $\alpha=-x-1$.

(c) $\log |F(z, \alpha)|$ is, as a function of $\alpha$, convex on the interval $(y-x-1$, $+\infty)$.

(d) $\log |F(z, \alpha)|$ has, as a function of $\alpha$, at most one inflection value on $(-x-1, y-x-1)$.

Statements (a), (b), and (c) follow at once from (2.9), (2.10), and (2.11) respectively. The zeros of $\partial^{2} \log |F(z, \alpha)| / \partial \alpha^{2}$ have been carefully investigated by Hille [5, p. 14] and (d) is included among his results. This may also be proven, though not so elegantly, by methods of $\$ 4$ to follow.

3. The summability method $\left[\Gamma(\alpha+\beta+1) C^{\alpha} \cdot C^{\beta}-\Gamma(\alpha+1) \Gamma(\beta+1) C^{\alpha+\beta}\right]$. Choose

$$
T(z)=\frac{C^{\alpha}(z) \cdot C^{\beta}(z)}{\Gamma(\alpha+1) \Gamma(\beta+1)}-\frac{C^{\alpha+\beta}(z)}{\Gamma(\alpha+\beta+1)} .
$$

From (1.9), we have

$$
T(z)=\frac{\alpha \beta}{(z+1)^{\alpha+\beta+1}}+O\left(|z+1|^{-\alpha-\beta-2}\right)
$$

uniformly on $R_{\boldsymbol{\epsilon}}$. Thus by statement (A), $\S 1$, and the succeeding comment, 
we have: If $\Re(\alpha+\beta)>-2$ and none of $\alpha, \beta, \alpha+\beta=-1$, then

$$
\left[\Gamma(\alpha+\beta+1) C^{\alpha} C^{\beta}-\Gamma(\alpha+1) \Gamma(\beta+1) C^{\alpha+\beta}\right] \supset C^{\alpha+\beta+1} .
$$

For real orders, the following contains a partial converse to this result:

II. At least, if $\alpha, \beta, \alpha+\beta$ are real and exceed -1 , and neither $\alpha$ nor $\beta$ is zero, then the summability method

$$
\begin{aligned}
& {\left[\Gamma(\alpha+\beta+1) C^{\alpha} \cdot C^{\beta}-\Gamma(\alpha+1) \Gamma(\beta+1) C^{\alpha+\beta}\right] } \\
& \cdot[\Gamma(\alpha+\beta+1)-\Gamma(\alpha+1) \Gamma(\beta+1)]^{-1}
\end{aligned}
$$

is equivalent to $C^{\alpha+\beta+1}$.

Proof. Assume the hypotheses true. From statement (B), §1, and the succeeding comment, it is seen that we need only prove $T(z) \neq 0$ on $R_{0}$. Hereafter suppose $z$ satisfies this last condition.

Suppose $z$ is real. We have

$$
\begin{aligned}
\frac{\alpha}{\Gamma(\alpha+1)} \int_{0}^{1}(1-x)^{\alpha-1}\left(1-x^{\beta}\right) x^{z} d x & \\
= & \frac{\Gamma(z+1)}{\Gamma(\alpha+z+1)}-\frac{\Gamma(\beta+z+1)}{\Gamma(\alpha+\beta+z+1)} .
\end{aligned}
$$

A comparison of this with (3.1) shows $T(z) \neq 0$.

Now consider $z=x+i y, y \neq 0$. We may write

$$
T(z)=\frac{\Gamma(z+1)}{\Gamma(\alpha+z+1)}\left[1-\frac{\Gamma(\alpha+z+1) \Gamma(\beta+z+1)}{\Gamma(z+1) \Gamma(\alpha+\beta+z+1)}\right] .
$$

Call the subtracted function in the brackets $G(z, \alpha, \beta)$. We must show $G(z, \alpha, \beta) \neq 1$. Since this function is symmetrical in $\alpha$ and $\beta$, it is no restriction to suppose $\alpha<\beta$. The desired proof is divided into three parts:

Part 1. $\alpha>0$. Using (2.4), we can write

$$
G(z, \alpha, \beta)=F(z, \alpha) F(z, \beta) / F(z, \alpha+\beta)
$$

so that

(3.6) $\log |G(z, \alpha, \beta)|=\log |F(z, \alpha)|+\log |F(z, \beta)|-\log |F(z, \alpha+\beta)|$.

Now $\log |F(z, 0)|=0$, and by Lemma $1, \log |F(z, \alpha)|$ is a convex function of $\alpha$ on $(0,+\infty)$. Therefore (3.6) is definitely negative and therefore (3.5) cannot be 1 .

Part 2. $-1<\alpha<0, \beta \geqq 1$. By Lemma $1, \log |F(z, \alpha)|$ is seen to be an increasing function on $(1,+\infty)$. Hence $\log |F(z, \beta)|>\log |F(z, \alpha+\beta)|$. Furthermore, if we again refer to the lemma, $\log |F(z, \alpha)|$ is positive. Thus $|G(z, \alpha, \beta)|$ exceeds 1 .

Part 3. $-1<\alpha<0,-1<\beta<1$. With the aid of (2.7), we can write 


$$
G(z, \alpha, \beta)=\prod_{0}^{\infty}\left[\frac{(\alpha+\beta+z+1)(z+j+1)}{(\alpha+z+j+1)(\beta+z+j+1)}\right] .
$$

Let us suppose $\Im(z)>0$. Consider the Argand diagrams of the vectors

$$
\frac{\alpha+\beta+z+j+1}{\alpha+z+j+1}, \quad \frac{\beta+z+j+1}{z+j+1} .
$$

It is clear that, according as $\beta>0$ or $\beta<0$, the argument of the first vector is negative and less than that of the second, or is positive and exceeds that of the second. This implies that the argument of (3.7) is not 0 . It is clear from the diagram that, as $|\beta|<1$,

$$
\arg |G(z, \alpha, \beta)|<\sum_{0}^{\infty}\left|\arg \frac{(\alpha+\beta+z+j+1)}{(\alpha+z+j+1)}\right| .
$$

Now the right-hand side of (3.8) is dominated by the greater of $\arg (\alpha+z+1)$ and arg $(\alpha+\beta+z+1)$. Since $(\alpha+1),(\alpha+\beta+1)$ are positive, it follows that neither of these exceeds $\pi / 2$. A similar result is found in the case $\Im(z)<0$. Thus, generally,

$$
0<|\arg G(z, \alpha, \beta)|<\pi / 2 .
$$

Therefore $G(z, \alpha, \beta) \neq 1$.

4. A result for Riesz summability methods. We need the following important results of Rogosinski [8]: Let $\phi(x)$ be constant on the exterior of the closed interval $(0,1)$, and of b.v. thereon. Let $s(x)$ be Borel-measurable (admissible). Write

$$
L(\omega)=\int_{0}^{\omega} s(x) d \phi(x / \omega) .
$$

To say that $s(x)$ is $H(\phi)$-convergent to $s$ means that $\lim _{\omega \rightarrow \infty} L(\omega)=s$. Let $T_{1}(z), T_{2}(z)$ be the Mellin transforms of $\phi_{1}(x), \phi_{2}(x)$ respectively; then

$$
\begin{aligned}
& H\left(T_{1}\right) \supset H\left(T_{2}\right) \text { implies } \quad H\left(\phi_{1}\right) \supset H\left(\phi_{2}\right)\left({ }^{3}\right) ; \\
& H\left(T_{1}\right) \cong H\left(T_{2}\right) \text { implies } \quad H\left(\phi_{1}\right) \cong H\left(\phi_{2}\right) .
\end{aligned}
$$

In particular, let $\lambda$ : $0<\lambda_{0}<\lambda_{1}<\cdots$ be a diverging sequence. Define the " $\lambda$-step function," $S^{\lambda}(x)$, to be that function which has value $s_{n}$ if $\lambda_{n} \leqq x<\lambda_{n+1}$, and value 0 if $x<\lambda_{0}$. Clearly this function is admissible. Write

$$
C_{\alpha}^{\lambda}(\omega)=-\int_{0}^{\omega} S^{\lambda}(x) d\left(1-\frac{x}{\omega}\right)^{\alpha} \quad(\Re(\alpha)>0) .
$$

The sequence $\left\{s_{n}\right\}$ will be called $C_{\alpha}^{\lambda}$-convergent (convergent by the Riesz

(8) In fact, if $H\left(\phi_{2}\right)-\lim s(x)=s$, then $H\left(\phi_{1}\right)-\lim s(x)=\left(T_{1}(0) / T_{2}(0)\right) s$. 
summability of order $\alpha$, and type $\lambda$ [3]) to $s$ if $\lim _{\omega \rightarrow \infty} C_{\alpha}^{\lambda}(\omega)=s$.

Let $r$ be any positive number. Write $\lambda^{r}=\left\{\lambda_{n}^{r}\right\}$. The following are true:

IIIA. Suppose $\Re(\alpha)>0, r>0$. Let $\left\{s_{n}\right\}$ be any sequence. If $\left\{s_{n}\right\}$ is $C_{\alpha+1^{-}}^{\lambda}$ convergent to $s$, then

$$
\lim _{\omega \rightarrow \infty}\left[C_{\alpha}^{\lambda}(\omega)-r^{\alpha} C_{\alpha}^{\left(\lambda^{r}\right)}\left(\omega^{1 / r}\right)\right]=\left[1-r^{\alpha}\right] s
$$

The real converse is:

IIIB. Suppose $r>0, \neq 1$. Let $\left\{s_{n}\right\}$ be any sequence. If $\alpha$ is a positive number, then (4.2) implies that $\left\{s_{n}\right\}$ is $C_{\alpha+1}^{\lambda}$-convergent to s.

Proof of IIIA. Consider

$$
T(z)=\alpha \int_{0}^{1}\left\{(1-x)^{\alpha-1}-r^{\alpha-1}\left(1-x^{1 / r}\right)^{\alpha-1} x^{1 / r-1}\right\} x^{z} d x .
$$

We find by integration,

$$
\frac{T(z)}{\Gamma(\alpha+1)}=\frac{\Gamma(z+1)}{\Gamma(\alpha+z+1)}-\frac{r^{\alpha} \Gamma(r z+1)}{\Gamma(\alpha+r z+1)} .
$$

As an application of (1.9), we have

$$
\frac{T(z)}{\Gamma(\alpha+1)}=\frac{\alpha(\alpha+1)}{2}\left(\frac{1-r}{2}\right)(z+1)^{-\alpha-1}+O\left(|z+1|^{-\alpha-2}\right) .
$$

Thus by statement (A), $\S 1$, and succeeding comment, it is seen that $H(T)$ $\supset C^{\alpha+1}$.

Write

$$
\phi(x)=\alpha \int_{0}^{x}\left[(1-t)^{\alpha-1}-r^{\alpha-1}\left(1-t^{1 / r}\right)^{\alpha-1} t^{1 / r-1}\right] d t
$$

and note that $\phi(1)-\phi(0)=1-r^{\alpha}$. Denote

$$
L_{\alpha}^{\lambda}(\omega)=\int_{0}^{1} S^{\lambda}(\omega x) d \phi(x) .
$$

It follows at once from (a) that if $\left\{s_{n}\right\}$ is $C_{\alpha+1}^{\lambda}$-convergent to $s$, then

$$
\lim _{\omega \rightarrow \infty} L_{\alpha}^{\lambda}(\omega)=\left[1-r^{\alpha}\right] s .
$$

Equation (4.7) can now be written

$$
L_{\alpha}^{\lambda}(\omega)=\alpha \int_{0}^{1} S^{\lambda}(x \omega)(1-x)^{\alpha-1} d x-\alpha r^{\alpha} \int_{0}^{1} S^{\lambda}(x \omega)\left(1-x^{1 / r}\right) \frac{x^{1 / r-1}}{r} d x .
$$

As $S^{\lambda}(x \omega)=S^{\left(\lambda^{r}\right)}\left(x \omega^{1 / r}\right)$, we have 


$$
L_{\alpha}^{\lambda}(\omega)=C_{\alpha}^{\lambda}(\omega)-r^{\alpha} C_{\alpha}^{\left(\lambda^{r}\right)}\left(\omega^{1 / r}\right) .
$$

Thus (4.2a) may be written as (4.2), and IIIA is established.

Proof of IIIB. From (a), and statement (B), $\$ 1$, it is seen that we need only prove $T(z) \neq 0$ on $R_{0}$, under the given conditions. Suppose the latter to hold throughout.

If we temporarily display the dependence of $T(z)$ on $\alpha$ and $r$, we find

$$
T(z, \alpha, r)=\left(-r^{\alpha}\right) T\left(z r, \alpha, r^{-1}\right) .
$$

This is easily proven with the aid of (4.4).

Denote

$$
K(z, \alpha, r)=r^{\alpha} \Gamma(r z+1) \Gamma(\alpha+z+1) / \Gamma(z+1) \Gamma(\alpha+r z+1) .
$$

From (4.4), it is seen that it is sufficient for us to prove $K(z, \alpha, r) \neq 1$. From (1.9) and (4.5), we have

$$
K(z, \alpha, r)=1+\frac{\alpha(\alpha+1)}{2}\left(\frac{r-1}{r}\right)\left(\frac{1}{z+1}\right)+O\left(|z+1|^{-2}\right) .
$$

Expressed as an infinite product

$$
K(z, \alpha, r)=r^{\alpha} \prod_{0}^{\infty}\left[\frac{(\alpha+r z+j+1)(z+j+1)}{(\alpha+z+j+1)(r z+j+1)}\right] .
$$

Because of (4.8), there will be no loss of generality if, from now on, we suppose $r>1$. Under this condition it will be shown that for all positive values of $\alpha,|K(z, \alpha, r)|>1$ on $R_{0}$.

We first prove this for the special case $z=i y$. As $K(0, \alpha, r)=r^{\alpha}$ which exceeds 1 if $\alpha>0$, it will be further supposed that $y \neq 0$.

From (4.11), we find

$$
\begin{aligned}
& \log |K(i y, \alpha, r)|^{2}=\alpha \log \left(r^{2}\right) \\
& +\sum_{0}^{\infty}\left[\log \left\{\frac{(\alpha+j+1)^{2}+y^{2} r^{2}}{(\alpha+j+1)^{2}+y^{2}}\right\}+\log \left\{\frac{(j+1)^{2}+y^{2}}{(j+1)^{2}+r^{2} y^{2}}\right\}\right] .
\end{aligned}
$$

For simplicity in notation, $\left(r^{2} y^{2}\right)^{1 / 2}$ will be denoted by $u$, and $\left(1 / y^{2}\right)$ $\cdot \partial \log |K(i y, \alpha, r)|^{2} / \partial\left(r^{2}\right)$ by $L\left(\alpha, r^{2} y^{2}\right)$. Thus

$$
\begin{aligned}
L(\alpha, u) & =\frac{\alpha}{u^{2}}-\sum_{0}^{\infty}\left[\frac{1}{(\alpha+j+1)^{2}+u^{2}}-\frac{1}{(j+1)^{2}+u^{2}}\right] \\
\frac{\partial L(\alpha, u)}{\partial \alpha} & =\frac{1}{u^{2}}-2 \sum_{0}^{\infty}\left[\frac{\alpha+j+1}{\left[(\alpha+j+1)^{2}+u^{2}\right]^{2}}\right] \\
\frac{\partial^{2} L(\alpha, u)}{\partial \alpha^{2}} & =2 \sum_{0}^{\infty}\left[\frac{3(\alpha+j+1)^{2}-u^{2}}{\left[(\alpha+j+1)^{2}+u^{2}\right]^{3}}\right]
\end{aligned}
$$


The following are true of the function $L(\alpha, u)$ :

(a) $L(0, u)=L(-1, u)=0$.

(b) $L_{\alpha}^{\prime}(0, u)=L_{\alpha}^{\prime}(-1, u)$.

(c) As a function of $\alpha, L(\alpha, u)$ is convex on $\left(\left(u / 3^{1 / 2}\right)-1,+\infty\right)$.

(d) As a function of $\alpha, L(\alpha, u)$ possesses at most one inflection value on $\left(-1,\left(u / 3^{1 / 2}-1\right)\right.$.

The first three of these statements follow directly from (4.13), (4.14), and (4.15) respectively. The proof of (d) is momentarily postponed.

The following conclusions are drawn from (a)-(d): The function $L(\alpha, u)$ is, as a function of $\alpha$, concave at $\alpha=-1$; it changes from concave to convex at some interior value of $(-1,0)$, and it is convex thereafter. It has on the interior of $(-1,0)$ precisely one zero, and on the open interval $(0,+\infty)$ is a positive function.

As a consequence of the last italicized statement, if $\alpha>0, \log |K(i y, \alpha, r)|$ is an increasing function of $r$. From (4.12), $\log \mid K(i y, \alpha, 1)=0$. Therefore $\log |K(i y, \alpha, r)|$ is positive if $\alpha>0$. Thus $|K(i y, \alpha, r)|>1$ on the line $\Re_{0}(z)=0$.

Referring to (4.10), it is clear that there exists some value $\rho_{0}$ such that, if $\rho>\rho_{0}$, then $|K(z, \alpha, r)|>1$ provided $\Re(z) \geqq 0$ and $|z|=\rho$. Using this together with the conclusion of the last paragraph, the minimum modulus principle tells us that $|K(z, \alpha, r)|>1$ on $R_{0}$. Thus IIIB is established.

Proof of (d). We shall use the notation, $f^{+}(t)=\max \left(f(t, 0)\left(f^{-}(t)\right.\right.$ $=\min (f(t), 0))$. By $f_{u}(t)$, we shall mean $f(u+j)$, where $j$ is the integral part of $(t-u)$.

Suppose $f(t) \geqq 0$ on the interval $(a,+\infty)$. Let $f(t)$ be nondecreasing on the closed interval $(a, M)$ and nonincreasing on the closed interval $(M,+\infty)$. Suppose that $f(t)$ is integrable on $(a,+\infty)$. The function has then the following simple properties:

$\left(\mathrm{P}_{1}\right)$

$$
\left|\int_{b}^{+\infty} f_{b}(t) d t-\int_{b}^{+\infty} f(t) d t\right| \leqq f(M) \quad(a \leqq b) .
$$

$\left(\mathrm{P}_{2}\right)$ Suppose $a \leqq b<M<c$. If $f(b) \geqq f(c)$, then

$$
\int_{c}^{+\infty} f_{b}(t) d t \geqq \int_{c}^{+\infty} f(t) d t
$$

$\left(\mathrm{P}_{3}\right)$ Suppose $M<b<+\infty$. If $f(t)$ is convex on $(b,+\infty)$, then

$$
\int_{b}^{+\infty} f_{b}(t) d t-\int^{\infty} f(t) d t \geqq f(b) / 2 .
$$

Proceeding now with our proof, we have upon differentiation of (4.15)

$$
\frac{1}{24} \frac{\partial^{3} L(\alpha, u)}{\partial \alpha^{3}}=\sum_{0}^{\infty} \frac{(\alpha+j+1)\left[u^{2}-(\alpha+j+1)^{2}\right]}{\left[(\alpha+j+1)^{2}+u^{2}\right]^{4}} .
$$


Denote

$$
\begin{aligned}
& g(t)=\left(3 t^{2}-u^{2}\right) /\left(t^{2}+u^{2}\right)^{3} \\
& \phi(t)=t\left(u^{2}-t^{2}\right) /\left(t^{2}+u^{2}\right)^{4}
\end{aligned}
$$

$$
u) / \partial \alpha^{2} \text {. We have then }
$$

$$
\begin{aligned}
G(\alpha, u) & =2 \int_{\alpha+1}^{+\infty} g_{\alpha+1}(t) d t, \\
\frac{\partial G(\alpha, u)}{\partial \alpha} & =24 \int_{\alpha+1}^{+\infty} \phi_{\alpha+1}(t) d t .
\end{aligned}
$$

The proof of (d) is based on the following statements:

(i) If $u \leqq 3^{1 / 2}, 0 \leqq \alpha+1<u / 4\left(3^{1 / 2}\right)$, then $G(\alpha, u)<0$.

Proof. By double differentiation of $\int_{0}^{+\infty} y d x /\left(x^{2}+y^{2}\right)^{2}$, or by a contour integration, we find

$$
\int_{0}^{+\infty} g(t) d t=0
$$

Computation yields

$$
g(\alpha+1) \leqq g\left(u / 4\left(3^{1 / 2}\right)\right)<\left(-0.88 u^{-4}\right)
$$

and

$$
\int_{u / 3^{1 / 2}}^{+\infty} g(t) d t=-\int_{0}^{u / 3^{1 / 2}} g(t) d t<\left(0.61 u^{-4}\right)\left(u / 3^{1 / 2}\right) .
$$

The greatest value of $g(t)$ is $1 / 4 u^{4}$, which occurs at $t=u$. It follows from $\left(\mathrm{P}_{1}\right)$ and (4.21) that

$$
\int_{0}^{+\infty} g_{\alpha+1}^{+}(t) d t<\left(0.61 u^{-4}\right) /\left(u / 3^{1 / 2}\right)+1 / 4 u^{4} .
$$

From the second hypothesis of (i) together with (4.20) we can also write

$$
\int_{0}^{+\infty} g_{\alpha+1}^{-}(t) d t \leqq\left(-0.88 u^{-4}\right) \text {. }
$$

Thus it is seen that

$$
G(\alpha, u)=2 \int_{0}^{+\infty} g_{\alpha+1}(t) d t<0 .
$$

(ii) If $u \geqq 0.58$ and there is $a$ value of $j$ such that $u / 4\left(3^{1 / 2}\right) \leqq \alpha+j+1$ $\leqq u / 3^{1 / 2}$, then $\partial G(\alpha, u) / \partial \alpha<0$.

Proof. Let $M(m)$ be the positive value at which $\phi(t)$ achieves its maximum 
(minimum) value. Computation yields

$$
\begin{gathered}
l: \int_{u / 3^{1 / 2}}^{+\infty} \phi(t) d t=0, \quad m: \phi\left(u / 3^{1 / 2}\right)=1 / 8 u^{5}, \\
n: \phi^{\prime \prime}\left(u / 3^{1 / 2}\right)=0, \\
o: \phi(m)=\left(-0.0177 u^{-5}\right), \quad p: \phi\left(u / 4\left(3^{1 / 2}\right)\right)=0.130 u^{-5} .
\end{gathered}
$$

If $M \leqq \alpha+j+1 \leqq u / 3^{1 / 2}$, then generally

$$
\begin{aligned}
\int_{\alpha+1}^{+\infty} \phi_{\alpha+1}^{+}(t) d t & >\int_{u / 3^{1 / 2}}^{+\infty} \phi_{u / 3^{1 / 2}}^{+}(t) d t>\int_{u / 3^{1 / 2}}^{+\infty} \phi(t) d t+\phi\left(u / 3^{1 / 2}\right) / 2 \\
& >\left|\int_{\alpha+1}^{+\infty} \phi^{-}(t) d t+\phi(m)\right|>\left|\int_{\alpha+1}^{+\infty} \phi_{\alpha+1}^{-}(t) d t\right| .
\end{aligned}
$$

The first of these inequalities is obvious; the second is established with the aid of $\left(\mathrm{P}_{3}\right)$; the third is true because $\phi\left(u / 3^{1 / 2}\right) / 2>\phi(m)$, and the last is a modified application of $\left(\mathrm{P}_{1}\right)$.

On the other hand, suppose $u / 4\left(3^{1 / 2}\right) \leqq \alpha+1<M$. Noting that $\phi\left(u / 3^{1 / 2}\right)$ $<\phi\left(u / 4\left(3^{1 / 2}\right)\right)$, we have

$$
\begin{aligned}
\int_{\alpha+1}^{+\infty} \phi_{\alpha+1}(t) d t> & \phi\left(u\left(3^{1 / 2}\right)\right)\left[u / 3^{1 / 2}-M\right]+\int_{u / 3^{1 / 2}}^{+\infty} \phi^{+}(t) d t \\
& +\int_{u\left(3^{1 / 2}\right)}^{+\infty} \phi^{-}(t) d t+\phi(m) .
\end{aligned}
$$

But $\int_{u, 3^{1 / 2}}^{+\infty} \phi(t) d t=0$, so

$$
\int_{\alpha+1}^{+\infty} \phi_{\alpha+1}(t) d t>\phi\left(u / 3^{1 / 2}\right)\left[u / 3^{1 / 2}-M\right]+\phi(m) .
$$

The right side of (4.28) is zero at $u=0.57$ (approximately); therefore, if $u \geqq 0.58$, it is true that $\partial G(\alpha, u) / \partial \alpha>0$. This with (4.26) proves (ii).

(iii) Suppose $u \leqq 2.8$. If there exists a value $j$, such that $u / 5\left(3^{1 / 2}\right) \leqq \alpha+j+1$ $\leqq 6 u / 5\left(3^{1 / 2}\right)$, then $\partial G(\alpha, u) / \partial \alpha>0$.

Proof. Computation yields

$$
\begin{gathered}
l: \quad \int_{u}^{+\infty} \phi(t) d t=1 / 48 u^{4}, \\
m: \phi\left(u / 5\left(3^{1 / 2}\right)\right)=0.111 u^{-5}, \quad n: \phi\left(6 u / 5\left(3^{1 / 2}\right)\right)=0.0769 u^{-5} .
\end{gathered}
$$

Certainly, then,

$$
\int_{\alpha+1}^{+\infty} \phi_{\alpha+1}^{+}(t) d t>\phi\left(6 u / 5\left(3^{1 / 2}\right)\right)
$$


The inequality

$$
\phi\left(6 u / 5\left(3^{1 / 2}\right)\right)+\int_{u}^{+\infty} \phi(t) d t+\phi(m)>0
$$

has, using the values (4.25 0 ) and (4.29), the approximate solution $u<2.9$. Thus if $u \leqq 2.8$, it follows from (4.30) that $\partial G(\alpha, u) / \partial u>0$.

Recalling the hypothesis $0 \leqq \alpha+1 \leqq u / 3^{1 / 2}$, the truth of (d) is now directly implied by the following:

(1) If $u \geqq\left(3 / 4\left(3^{1 / 2}\right)\right)^{-1}$, there exists a $j$ such that the hypotheses of (ii) are satisfied. Hence $\partial G(\alpha, u) / \partial \alpha>0$.

(2) If $3^{1 / 2} \leqq u \leqq\left(3 / 4\left(3^{1 / 2}\right)\right)^{-1}$, there exists a $j$ such that $u / 5\left(3^{1 / 2}\right) \leqq \alpha+j$ $+1 \leqq 6 u / 5\left(3^{1 / 2}\right)$. Thus by (iii), $\partial G(\alpha, u) / \partial \alpha>0$.

(3) Suppose $u<3^{1 / 2}$. Then a direct application of (i) and (iii) shows that $G(\alpha, u)$ is negative if $0 \leqq \alpha+1 \leqq u / 4\left(3^{1 / 2}\right)$, and is otherwise an increasing function.

Thus, in any event, $G(\alpha, u)$ has, at most, one zero if $0 \leqq \alpha+1<u / 3^{1 / 2}$. This proves (d).

In closing, I should like to thank the reviewer of the paper as it was originally presented, for a very thorough and extremely helpful criticism.

\section{BIBLIOGRAPHY}

1. H. L. Garabedian, E. Hille, and H. S. Wall, Formulations of the Hausdorff inclusion problem, Duke Math. J. vol. 8 (1941) pp. 193-213.

2. G. H. Hardy, Divergent series, Oxford, Clarendon, 1949, chap. 11.

3. G. H. Hardy and M. Riesz, The general theory of Dirichlet series, Cambridge Tracts in Mathematics and Physics, no. 18, 1915.

4. F. Hausdorff, Summationsmethoden und Momentfolgen (I), Math. Zeit. vol. 9 (1921) pp. 74-109.

5. E. Hille, On the logarithmic derivative of the gamma function, Det Kegl. Danske Videnskabernes Selskab vol. 8 (1927) pp. 3-58.

6. R. E. A. C. Paley and N. Wiener, Fourier transforms in the complex domain, Amer. Math. Soc. Colloquium Publications, vol. 19, New York, 1934, chap. 1.

7. W. W. Rogosinski, On Hausdorff's methods of summability (I), Proc. Cambridge Philos. Soc. vol. 38 (1942) pp. 166-192.

8. - On Hausdorff's methods of summability (II), Proc. Cambridge Philos. Soc. vol. 38 (1942) pp. 344-363.

9. H. S. Wall, Analytic theory of continued fractions, New York, Van Nostrand, 1948, chaps. $14,16$.

10. E. Whittaker and G. Watson, $A$ course of modern analysis, New York, Macmillan, 1945, pp. 278-279.

11. D. V. Widder, The Laplace transform, Princeton University Press, 1941, chap. 3.

\section{University of Alabama,}

University, Ala. 